\title{
Extended-schedule dose-dense temozolomide in refractory gliomas
}

\author{
A. Berrocal · P. Perez Segura $\cdot$ M. Gil · C. Balaña $\cdot$ \\ J. Garcia Lopez $\cdot$ R. Yaya $\cdot$ J. Rodríguez $\cdot$ G. Reynes $\cdot$ \\ O. Gallego $\cdot$ L. Iglesias $\cdot$ GENOM Cooperative Group
}

Received: 21 May 2009/Accepted: 20 July 2009/Published online: 8 August 2009

(c) The Author(s) 2009. This article is published with open access at Springerlink.com

\begin{abstract}
This multicenter phase II study conducted by the Spanish Neuro-Oncology Group evaluated the activity of an extended, dose-dense temozolomide regimen in patients with temozolomide-refractory malignant glioma. Adult patients (at least 18 years of age) with WHO grade III or IV glioma and a Karnofsky Performance Status of 60 or higher were treated with temozolomide $\left(85 \mathrm{mg} / \mathrm{m}^{2} /\right.$ day $)$ for 21 consecutive days every 28-day cycle until disease progression or unacceptable toxicity. All patients had developed progressive disease either during or less than 3 months after completing previous temozolomide treatment. Forty-seven patients were treated with a median of 2 (range, 1-13) cycles of temozolomide. Before study entry, patients had received a median of 6 cycles of temozolomide: $39(83 \%)$ as part of initial therapy and $23(49 \%)$ as second-line therapy. Three patients $(6.4 \%)$ had a partial response with durations of $8.0,3.5$, and 3.2 months; 15
\end{abstract}

\footnotetext{
A. Berrocal ( $\square)$

Servicio de Oncologia Medica, Consorcio Hospital

General Universitario de Valencia, Avda Tres Cruces S/N,

46006 Valencia, Spain

e-mail: berrocal_alf@gva.es

P. Perez Segura

Hospital Clinico San Carlos, Madrid, Spain

M. Gil

Instituto Catalan de Oncologia Duran i Reynals,

Barcelona, Spain

C. Balaña

Hospital Germans Trias i Pujol, Badalona, Spain

J. Garcia Lopez

Hospital Ramon y Cajal, Madrid, Spain
}

patients (31.9\%) had stable disease with a median duration of 2.1 months, including 2 patients with stable disease (SD) for greater than 6 months (14 and 16 months). Median time to progression was 2 months, and median overall survival from study entry was 5.1 months. The 6-month progression-free survival rate was $16.7 \%$. The most common hematologic toxicities were lymphopenia, thrombocytopenia, and leukopenia. Lymphopenia occurred in $83 \%$ of patients and was grade 3 in $28 \%$, but no opportunistic infections occurred. In conclusion, this extended dosedense schedule of temozolomide appears to have modest activity in patients refractory to previous treatment with temozolomide and is associated with manageable toxicity.

Keywords Temozolomide $\cdot$ Activity $\cdot$ Refractory · Malignant glioma

\author{
R. Yaya \\ Instituto Valenciano de Oncologia, Valencia, Spain \\ J. Rodríguez \\ Hospital de Sondureta, Palma de Mallorca, Spain \\ G. Reynes \\ Hospital La Fe, Valencia, Spain \\ O. Gallego \\ Hospital Santa Creu i San Pau, Barcelona, Spain \\ L. Iglesias \\ Hospital Virgen del Rocio, Sevilla, Spain
}




\section{Introduction}

Malignant gliomas are aggressive tumors and, despite recent improvements in their treatment, almost always relapse after first-line treatment and remain incurable [1]. The majority of patients with high-grade gliomas receive temozolomide either as first-line treatment in combination with radiotherapy or at relapse, based on evidence from prospective trials demonstrating the clinical benefit of temozolomide in these settings [2, 3]. Temozolomide is an imidazotetrazine derivative that methylates the DNA at several sites including $\mathrm{N}^{7}$-guanine $(70 \%), \mathrm{N}^{3}$-adenine $(9 \%)$, or $\mathrm{O}^{6}$-guanine $(5 \%)$ [4]. However, only methylation at $\mathrm{O}^{6}$-guanine and $\mathrm{O}^{6}$-methylguanine DNA adducts appears to drive the cytotoxic activity of temozolomide [4, 5].

The DNA damage caused by temozolomide is repaired by $\mathrm{O}^{6}$-methylguanine methyltransferase (MGMT), which is consumed in the process [6]. Several studies have suggested that resistance to alkylating agents is primarily mediated by MGMT [6-8]. Furthermore, it has been demonstrated that temozolomide decreases MGMT activity in peripheral mononuclear cells in a schedule-dependent manner [9], and that protracted administration of temozolomide results in more extensive and sustained depletion of MGMT [10]. Finally, it has been demonstrated that depletion of MGMT enhances the cytotoxicity of temozolomide and other alkylating agents [11].

Based on these observations, it is reasonable to hypothesize that extended temozolomide administration may overcome resistance through MGMT depletion and restore sensitivity to temozolomide in patients with temozolomiderefractory malignant glioma. The present trial was designed by the Spanish Neuro-Oncology Group (GENOM) to test the activity of an extended, dose-dense temozolomide regimen $\left(85 \mathrm{mg} / \mathrm{m}^{2} \times 21\right.$ days every 28 days $)$ in patients who had progressed following first-line or second-line treatment with temozolomide. This dose was selected based on the results of a phase I trial in patients previously treated with temozolomide [12].

\section{Patients and methods}

\section{Patient eligibility}

Adult patients (at least 18 years of age) with histologically confirmed, temozolomide-refractory, WHO grade III or IV glioma and a Karnofsky Performance Status (KPS) of 60 or higher were included. Patients were eligible and considered refractory to temozolomide if they developed progressive disease during temozolomide treatment or less than 3 months after completing previous temozolomide treatment.
Adequate laboratory values were required as follows: absolute neutrophil count $\geq 1,500 / \mu \mathrm{l}$, platelet count $\geq 100,000 / \mu \mathrm{l}$, hemoglobin greater than $10 \mathrm{~g} / \mathrm{dl}$ or $100 \mathrm{~g} / \mathrm{l}$, blood urea nitrogen and serum creatinine less than 1.5 times the upper limit of laboratory normal, total and direct serum bilirubin less than 1.5 times the upper limit of laboratory normal, aspartate aminotransferase or alanine aminotransferase less than 3 times the upper limit of laboratory normal, and alkaline phosphatase less than 2 times the upper limit of laboratory normal. Patients were required to have been on a non-increasing corticosteroid dose for $\geq 72 \mathrm{~h}$ before baseline neuroimaging and study drug administration, and to have a life expectancy greater than 12 weeks. All patients were required to provide written informed consent.

Study design

This was a multicenter phase II trial conducted by the GENOM. Institutional review boards at each study center approved the study. The primary objective was to assess whether an extended temozolomide dosing schedule can overcome tumor resistance to the drug. Secondary objectives were response and tolerability. According to the 2-stage model of Simon et al. [13], the primary objective was considered not reached if no objective response was observed in the first 10 patients or if less than 3 responses were observed among 29 patients in the second stage. Sample size was estimated according to the Simon 2-stage phase II design $\left(\mathrm{P}_{0}=5 \%\right.$ response rate; $\mathrm{P}_{1}=20 \%$ response rate) [13]. To achieve an $(\alpha, \beta)$ error of $(0.05,0.2)$, a total of 29 patients were required, and to achieve an $(\alpha, \beta)$ error of $(0.05,0.1)$, a total of 43 patients were required.

\section{Treatment}

After a 4-week clearance period to allow recovery from toxicities, patients received temozolomide at a starting dose of $85 \mathrm{mg} / \mathrm{m}^{2} /$ day in a fasting state for 21 consecutive days every 28-day cycle. Temozolomide treatment was administered until disease progression (PD) or unacceptable toxicity occurred. Three dose levels were defined: $85 \mathrm{mg} / \mathrm{m}^{2}$, $75 \mathrm{mg} / \mathrm{m}^{2}$, and $65 \mathrm{mg} / \mathrm{m}^{2}$. If neutrophils were over $1500 / \mu \mathrm{l}$ and platelets over $100,000 / \mu \mathrm{l}$ patients were treated at the $85 \mathrm{mg} / \mathrm{m}^{2}$ dose; otherwise, treatment was delayed for 1 week to a maximum of 3 weeks until recovery. If neutrophils were below $500 / \mu$ l for 5 days, or platelets were below $25,000 / \mu \mathrm{l}$, or if the treatment delay was greater than 2 weeks, the dose was reduced to the next lower level. If nonhematologic toxicity of grade 3 or greater occurred, the dose was also reduced. Prophylactic antiemetic therapy with metoclopramide or 5-HT antagonist was administered to all patients. No Pneumocystis jirovecii pneumonia prophylaxis was administered. 
Assessment of response and safety

Response was assessed according to Macdonald's criteria [14]. Neurologic examination and steroid dose modification were recorded at each cycle. Neuroimaging with gadolinium-enhanced MRI was performed at least every 2 cycles or if PD was suspected based on neurologic symptoms. Complete response was defined as complete disappearance of all detectable tumors as determined by 2 gadolinium-enhanced MRI scans of the brain performed not less than 4 weeks apart. Adverse events were graded according to the National Cancer Institute Common Terminology Criteria for Adverse Events v3.0.

\section{Results}

A total of 47 patients were enrolled and received at least 1 cycle of temozolomide. All patients were assessable for response and safety. Baseline patient characteristics are shown in Table 1. Median time from the diagnosis to study

Table 1 Patient demographics and baseline clinical characteristics $(N=47)$

\begin{tabular}{ll}
\hline Mean age, years (range) & $50(20-77)$ \\
Sex, $n(\%)$ & $33(70)$ \\
Male & $14(30)$ \\
Female & \\
ECOG performance status, $n(\%)$ & $8(17)$ \\
0 & $20(43)$ \\
1 & $19(40)$ \\
2 & \\
Tumor histology, $n(\%)$ & $27(57)$ \\
Glioblastoma & $15(32)$ \\
Anaplastic astrocytoma & $3(6)$ \\
Anaplastic oligodendroglioma & $2(4)$ \\
Other & \\
Prior surgery, $n$ (\%) & $9(19)$ \\
Biopsy only & $20(43)$ \\
Partial resection & $17(36)$ \\
Gross total resection & $1(2)$ \\
Biopsy and partial resection & $n=47$ \\
Prior first-line chemotherapy, $n(\%)$ & $38(81)$ \\
Temozolomide & $4(9)$ \\
Carmustine & $4(9)$ \\
PCV & $1(2)$ \\
Temozolomide + cisplatin & $n=23$ \\
Prior second-line chemotherapy, $n(\%)$ & 21 \\
Temozolomide monotherapy & 2 \\
\hline
\end{tabular}

ECOG Eastern Cooperative Oncology Group; $P C V$ procarbazine, lomustine, and vincristine entry was 14 months (range 6-126 months). Before study entry, patients had received a median of 6 (range 1-21) cycles of temozolomide: 39 patients $(83 \%)$ as part of initial therapy and 23 patients (49\%) as second-line therapy. Thus, some patients had received temozolomide as part of both first- and second-line therapy. All patients had progressed during or within 3 months of completing standard-dose therapy and at least 6 months after completing chemoradiotherapy. No patients received dose-dense temozolomide before study entry. Anticonvulsant therapy was administered to 31 patients $(66 \%)$. The most frequently used anticonvulsants were phenytoin in 17 patients $(55 \%)$, valproic acid in 11 patients (36\%), gabapentin in 6 patients $(19 \%)$, and levetiracetam in 5 patients (16\%). Some patients received more than 1 anticonvulsant drug.

Patients received a median of 2 (range, 1-13) cycles of temozolomide on study, and a total of 168 treatment cycles were administered. Sixteen cycles were delayed in 7 patients as a result of hematologic toxicity in 3 patients, nonhematologic toxicity in 2 patients, and for administrative reasons in 2 patients. Only 3 cycles were dose reduced in 2 patients because of hematologic toxicity.

Among 47 treated patients, $3(6.4 \%)$ had a partial response (95\% confidence interval [CI]: $0.6 \%, 13.2 \%$ ), including 1 patient with oligodendroglioma who had received 3 previous lines of therapy, 1 patient with a glioblastoma who had received 2 previous lines of therapy, and 1 patient with a glioblastoma who had received 1 previous line of therapy. Duration of the responses was 8 , 3.2 , and 3.5 months, respectively. In addition, 15 patients (31.9\%) had stable disease (SD). Median duration of SD was 2.1 months, and 2 patients remained stable for greater than 6 months (14 and 16 months). One of 3 responders, 6 of 15 patients with SD, and 9 of 30 patients with PD on study had a response or SD lasting greater than 6 months during previous temozolomide therapy. All 3 patients who had a response or SD lasting greater than 6 months on study also had a response or SD lasting greater than 6 months during previous temozolomide therapy.

At a median follow up of 3.6 months, median time to progression was 2 months (95\% CI: 1.6, 2.4 months; Fig. 1) and median overall survival from study entry was 5.1 months (95\% CI: 3.7, 6.5 months; Fig. 2). The 6month progression-free survival (PFS) rates were $16.7 \%$ for the overall population, $0 \%$ among patients with glioblastoma, and $37.5 \%$ among patients with anaplastic astrocytoma or oligodendroglioma. The 6-month overall survival rate was $41.6 \%$ (95\% CI: $25.1 \%, 58.1 \%$ ).

The most common hematologic toxicities were lymphopenia, thrombocytopenia, and leukopenia (Table 2). Lymphopenia occurred in $83 \%$ of patients and was grade 3 in $28 \%$, but no opportunistic infections occurred. The most common nonhematologic adverse events or laboratory 


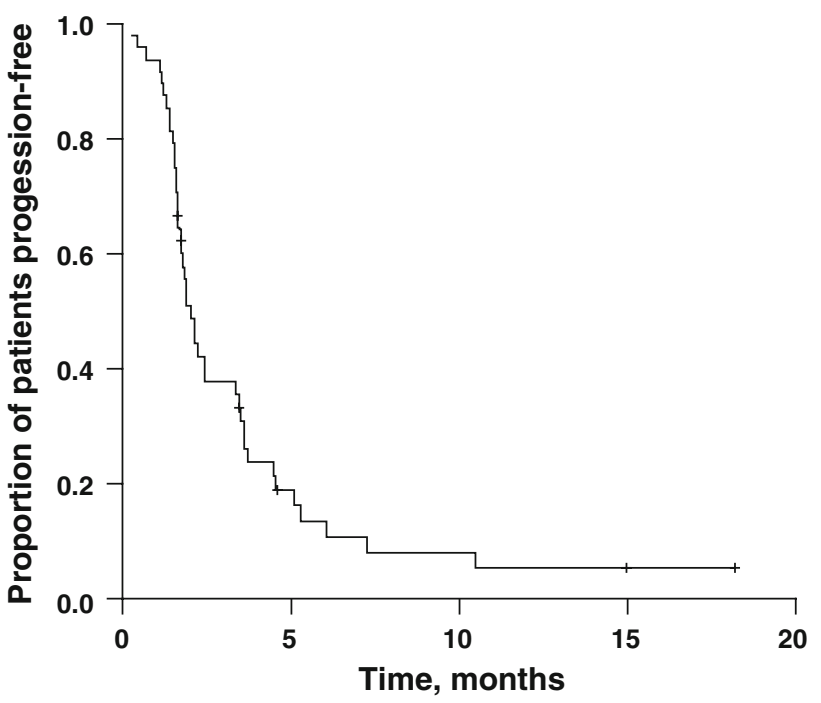

Fig. 1 Kaplan-Meier estimate of time to progression

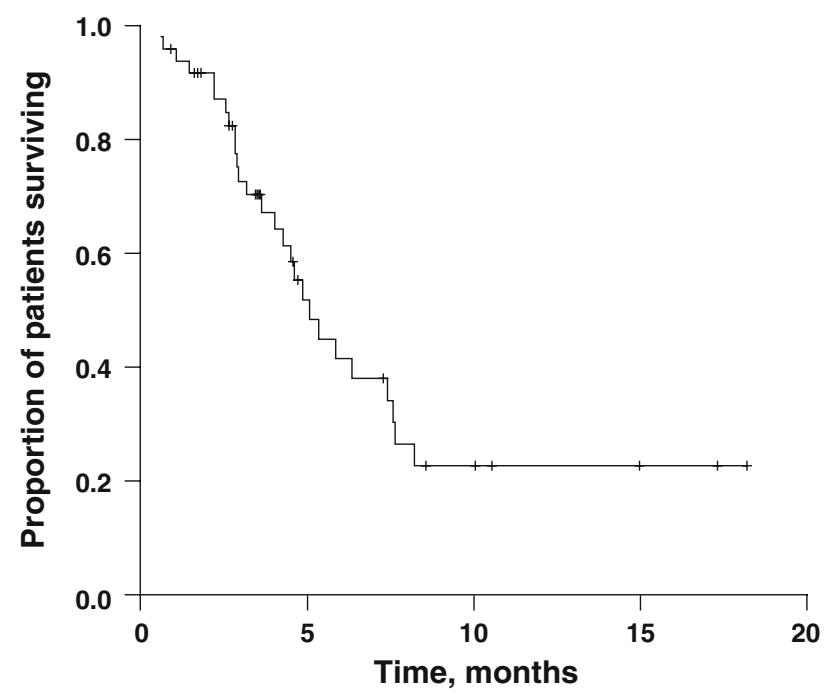

Fig. 2 Kaplan-Meier estimate of overall survival

Table 2 Hematologic toxicity by grade

\begin{tabular}{lccr}
\hline & \multicolumn{2}{l}{ Patients, $n(\%)$} \\
\cline { 2 - 4 } & Grade 1 or 2 & Grade 3 & Total \\
\hline Lymphopenia & $26(55)$ & $13(28)$ & $39(83)$ \\
Thrombocytopenia & $9(19)$ & $5(11)$ & $14(30)$ \\
Leukopenia & $13(28)$ & $1(2)$ & $14(30)$ \\
Anemia & $7(15)$ & $2(4)$ & $9(19)$ \\
Neutropenia & $8(17)$ & $1(2)$ & $9(19)$ \\
\hline
\end{tabular}

abnormalities were nausea/vomiting, asthenia, and mild asymptomatic transaminitis (Table 3). Most nonhematologic adverse events and laboratory abnormalities were mild to moderate in severity, and there was no grade 4 toxicity.
Table 3 Most common nonhematologic adverse events and laboratory abnormalities by grade

\begin{tabular}{lclc}
\hline & \multicolumn{2}{l}{ Patients, $n(\%)$} & \\
\cline { 2 - 4 } & Grade 1 or 2 & Grade 3 & Total \\
\hline Adverse events & & & \\
$\quad$ Nausea/vomiting & $12(26)$ & $1(2)$ & $13(28)$ \\
Asthenia & $8(17)$ & - & $8(17)$ \\
Diarrhea & $5(11)$ & - & $5(11)$ \\
Constipation & $2(4)$ & - & $2(4)$ \\
Mucositis & $2(4)$ & $1(2)$ & $3(6)$ \\
Laboratory abnormalities & & & \\
GPT & $24(51)$ & - & $24(51)$ \\
GOT & $9(19)$ & - & $9(19)$ \\
Creatinine & $6(13)$ & - & $6(13)$ \\
GGT & - & $1(2)$ & $1(2)$ \\
Bilirubin & $1(2)$ & - & $1(2$ \\
\hline
\end{tabular}

$G P T$ glutamic-pyruvic transaminase, $G O T$ glutamic-oxaloacetic transaminase, $G G T$ gamma-glutamyl transferase

\section{Discussion}

Since the publication of the pivotal phase III trial in 2005 demonstrating the superiority of combining radiotherapy with temozolomide compared with radiotherapy alone in newly diagnosed glioblastoma patients [8], temozolomide has become the standard of care for initial treatment of glioblastoma and is widely used for treatment of anaplastic glioma. Consequently, standard-dose temozolomide may no longer be the best treatment option at recurrence. A variety of options have been explored, including retreatment with temozolomide using a dose-dense schedule or treatment with nitrosoureas, irinotecan, bevacizumab, or a variety of novel combinations of these and other agents [15-19]. Carmustine has demonstrated only limited activity after temozolomide [18], whereas irinotecan, in combination with bevacizumab, carmustine, or thalidomide, has demonstrated promising activity in this setting [15-17, 19]. Presumably most high-grade gliomas are refractory to alkylating agents at recurrence following initial treatment with temozolomide. However, a recent report by Perry et al. [20] of interim data from the RESCUE study has shown that retreatment with continuous low-dose daily temozolomide resulted in favorable 6-month PFS rates in patients who progressed during or after first-line temozolomide. Other studies have also demonstrated the activity of dose-dense temozolomide regimens in this setting [21].

The results of the present study have further demonstrated that retreatment with temozolomide using an extended, dose-dense schedule can result in objective responses in patients previously exposed to temozolomide as part of their initial treatment. Although the observed 
6.3\% partial response rate and 6-month PFS rate were modest, patients enrolled in this study were pretreated with a median of 6 cycles of temozolomide, and many patients received extended treatment with up to 21 cycles of standard-dose temozolomide before study entry. Similarly, among patients enrolled in the RESCUE study, those who received prolonged maintenance therapy with standarddose temozolomide (beyond 6 cycles) had a low 6-month PFS rate $(9.5 \%)$ [20]. In addition, all patients entered the present study at least 6 months after completing previous radiotherapy. This suggests that the responses observed in the present study were not an artifact of pseudoprogression, but this cannot be completely ruled out.

It has been observed that patients who have prolonged SD after primary temozolomide treatment often have a prolonged response to retreatment with temozolomide [22, 23]. Consistent with these published reports, all patients who had a durable response or SD in the present study had previously had a durable response or SD to first- or secondline temozolomide. It should be noted, however, that objective responses and SD have been observed in patients who had a short duration of response to first-line temozolomide. Therefore, no patient should be excluded from retreatment with an extended temozolomide regimen based on the quality of their response to first-line temozolomide.

Compared with the standard 5-day dosing schedule every 28-day cycle, the extended dose-dense schedules result in approximately a 2-fold greater exposure to temozolomide and may have greater potential to deplete MGMT activity within tumor cells and overcome resistance [11]. The first report of an extended temozolomide regimen investigated a dose of $75 \mathrm{mg} / \mathrm{m}^{2} /$ day for 6 or 7 consecutive weeks [24]. This schedule resulted in a $41 \%$ response rate among 17 patients with recurrent glioma. Subsequent studies in glioblastoma investigating 2 different extended temozolomide schedules ( 7 consecutive days every 2 weeks, or 21 consecutive days every 28 days) showed that both schedules resulted in a significant and prolonged depletion of MGMT in peripheral blood mononuclear cells, which led to the hypothesis that these dose-dense regimens could potentially enhance antitumor activity [10]. Two phase II trials in patients with recurrent glioblastoma also demonstrated that these regimens are highly active $[23,25]$. In the first study conducted in temozolomide-naive patients, the 21-day continuous schedule resulted in a 9\% response rate and a 6month PFS rate of $30 \%$ [25]. In the second study, the majority of patients had not been previously exposed to temozolomide, and the alternating weekly regimen resulted in a response rate of $15 \%$ and a 6-month PFS rate of $44 \%$ [23]. Therefore, the fact that 2 of 27 (7\%) glioblastoma patients in the present study who were previously treated with temozolomide had an objective response supports the hypothesis that this dose-dense regimen may overcome resistance. One limitation of the present study is the lack of data on MGMT methylation status of these tumors. However, MGMT methylation status at initial diagnosis has limited value in the recurrent setting, particularly given the observation that methylation patterns within the tumor can change from initial diagnosis to recurrence.

Toxicity associated with extended, dose-dense temozolomide schedules has been reported to be greater than that associated with the conventional 5-day schedule and to consist mainly of lymphopenia [23, 25-28]. In the present study, grade 3 or higher lymphopenia occurred in nearly $30 \%$ of patients. However, no complications related to immunodeficiency such as Pneumocystis jirovecii pneumonia were reported, even in the absence of PCP prophylaxis. All toxicities were manageable, and few patients required treatment delays or dose reductions.

In conclusion, this extended dose-dense schedule of temozolomide appears to have activity, albeit modest, in patients who have failed previous treatment with temozolomide and is associated with manageable toxicity. These results suggest that extended schedules of temozolomide may be preferable to the standard temozolomide regimen and should be further evaluated in larger randomized studies with prospective stratification of patients by primary tumor type and extent of prior temozolomide therapy. The full results of the RESCUE study are eagerly awaited and may provide further insight regarding how best to use temozolomide in the recurrent setting.

Acknowledgments Financial support for medical editorial assistance was provided by Schering-Plough International. We thank Jeffrey Riegel, $\mathrm{PhD}$, ProEd Communications, Inc. ${ }^{\circledR}$, for his medical editorial assistance with this manuscript.

Open Access This article is distributed under the terms of the Creative Commons Attribution Noncommercial License which permits any noncommercial use, distribution, and reproduction in any medium, provided the original author(s) and source are credited.

\section{References}

1. Gilbert MR (2006) Advances in the treatment of primary brain tumors: dawn of a new era? Curr Oncol Rep 8:45-49

2. Stupp R, Mason WP, van den Bent MJ et al (2005) Radiotherapy plus concomitant and adjuvant temozolomide for glioblastoma. $\mathrm{N}$ Engl J Med 352:987-996

3. Yung WK, Albright RE, Olson J et al (2000) A phase II study of temozolomide vs. procarbazine in patients with glioblastoma multiforme at first relapse. Br J Cancer 83:588-593

4. Newlands ES, Stevens MF, Wedge SR, Wheelhouse RT, Brock C (1997) Temozolomide: a review of its discovery, chemical properties, pre-clinical development and clinical trials. Cancer Treat Rev 23:35-61

5. Tisdale MJ (1987) Antitumor imidazotetrazines-XV. Role of guanine O6 alkylation in the mechanism of cytotoxicity of imidazotetrazinones. Biochem Pharmacol 36:457-462 
6. Pegg AE (2000) Repair of O(6)-alkylguanine by alkyltransferases. Mutat Res 462:83-100

7. Bobola MS, Tseng SH, Blank A, Berger MS, Silber JR (1996) Role of O6-methylguanine-DNA methyltransferase in resistance of human brain tumor cell lines to the clinically relevant methylating agents temozolomide and streptozotocin. Clin Cancer Res 2:735-741

8. Hegi ME, Diserens A-C, Gorlia $\mathrm{T}$ et al (2005) MGMT gene silencing and benefit from temozolomide in glioblastoma. N Engl J Med 352:997-1003

9. Lee SM, Thatcher N, Crowther D, Margison GP (1994) Inactivation of O6-alkylguanine-DNA alkyltransferase in human peripheral blood mononuclear cells by temozolomide. Br J Cancer 69:452-456

10. Tolcher AW, Gerson SL, Denis L et al (2003) Marked inactivation of O6-alkylguanine-DNA alkyltransferase activity with protracted temozolomide schedules. Br J Cancer 88:1004-1011

11. Baer JC, Freeman AA, Newlands ES, Watson AJ, Rafferty JA, Margison GP (1993) Depletion of O6-alkylguanine-DNA alkyltransferase correlates with potentiation of temozolomide and CCNU toxicity in human tumour cells. Br J Cancer 67:1299-1302

12. Denis L, Tolcher A, Figueroa JA et al (2000) Protracted daily administration of temozolomide is feasible: a phase I and pharmacokinetic - pharmacodynamic study (abstract). Proc Am Soc Clin Oncol 19:202a Abstract 786

13. Simon R (1987) How large should a phase II trial of a new drug be? Cancer Treat Rep 71:1079-1085

14. Macdonald DR, Cascino TL, Schold SC, Cairncross JG (1990) Response criteria for phase II studies of supratentorial malignant glioma. J Clin Oncol 8:1277-1280

15. Brandes AA, Tosoni A, Basso U et al (2004) Second-line chemotherapy with irinotecan plus carmustine in glioblastoma recurrent or progressive after first-line temozolomide chemotherapy: a phase II study of the Gruppo Italiano Cooperativo di Neuro-Oncologia (GICNO) (published correction appears in $\mathbf{J}$ Clin Oncol 2005;23(1):248). J Clin Oncol 22:4779-4786

16. Puduvalli VK, Giglio P, Groves MD et al (2008) Phase II trial of irinotecan and thalidomide in adults with recurrent glioblastoma multiforme. Neuro Oncol 10:216-222

17. Reardon DA, Quinn JA, Rich JN et al (2004) Phase 2 trial of BCNU plus irinotecan in adults with malignant glioma. Neuro Oncol 6:134-144
18. Rosenthal MA, Ashley DL, Cher L (2004) BCNU as second line therapy for recurrent high-grade glioma previously treated with temozolomide. J Clin Neurosci 11:374-375

19. Vredenburgh JJ, Desjardins A, Herndon JEII et al (2007) Bevacizumab plus irinotecan in recurrent glioblastoma multiforme. J Clin Oncol 25:4722-4729

20. Perry JR, Mason WP, Belanger K et al (2008) The temozolomide RESCUE study: a phase II trial of continuous (28/28) doseintense temozolomide (TMZ) after progression on conventional $5 / 28$ day TMZ in patients with recurrent malignant glioma (abstract). J Clin Oncol 26(Suppl):91s (abstract 2010)

21. Wick A, Pascher C, Wick W et al (2009, Epub ahead of print) Rechallenge with temozolomide in patients with recurrent gliomas. J Neurol

22. Franceschi E, Omuro AM, Lassman AB, Demopoulos A, Nolan C, Abrey LE (2005) Salvage temozolomide for prior temozolomide responders. Cancer 104:2473-2476

23. Wick A, Felsberg J, Steinbach JP et al (2007) Efficacy and tolerability of temozolomide in an alternating weekly regimen in patients with recurrent glioma. J Clin Oncol 25:3357-3361

24. Brock CS, Newlands ES, Wedge SR et al (1998) Phase I trial of temozolomide using an extended continuous oral schedule. Cancer Res 58:4363-4367

25. Brandes AA, Tosoni A, Cavallo G et al (2006) Temozolomide 3 weeks on and 1 week off as first-line therapy for recurrent glioblastoma: phase II study from gruppo italiano cooperativo di neuro-oncologia (GICNO). Br J Cancer 95:1155-1160

26. Wick W, Weller M (2005) How lymphotoxic is dose-intensified temozolomide? The glioblastoma experience. J Clin Oncol 23:4235-4236

27. Chinot OL, Barrie M, Fuentes S et al (2007) Correlation between $\mathrm{O}^{6}$-methylguanine-DNA methyltransferase and survival in inoperable newly diagnosed glioblastoma patients treated with neoadjuvant temozolomide. J Clin Oncol 25:1470-1475

28. Neyns B, Chaskis C, Joosens E et al (2008) A multicenter cohort study of dose-dense temozolomide ( 21 of 28 days) for the treatment of recurrent anaplastic astrocytoma or oligoastrocytoma. Cancer Invest 26:269-277 